\title{
RIFLETTERE SULLA VITA MORALE CRISTIANA. LA PROPOSTA MORALE DI DOMENICO CAPONE 50 ANNI DOPO VATICANUM II
}

DOI: http://dx.doi.org/10.12775/TiCz.2015.048

Mostrare la grandezza della vocazione dei credenti in Cristo è il compito indicato dal Vaticano II alla teologia morale. Essa deve quindi aiutare l'uomo a comprendere la grandezza della sua vocazione e della libera e responsabile risposta a tale chiamata. Cinquant'anni dopo l'inizio del Concilio sarà molto utile rivedere l'appassionante percorso teologico di Domenico Capone - gran protagonista e continuatore della tradizione teologico morale dell'Accademia Alfonsiana - e della sua proposta morale attuale per l'uomo del XXI secolo. La sua intuizione nel riflettere sull'uomo, sullagire della persona umana e sulla vita morale del cristiano, che ha tracciato la via del rinnovamento della teologia morale, si è rivelata profetica rispetto alla tradizione morale della Chiesa di oggi.

* Ks. dr Artur Niemira - prezbiter diecezji włocławskiej, absolwent Papieskiego Uniwersytetu Gregoriańskiego w Rzymie. Prowadzi zajęcia z teologii moralnej w Wyższym Seminarium Duchownym we Włocławku oraz na Wydziale Teologicznym UMK w Toruniu, od 2004 r. kanclerz Kurii Diecezjalnej we Włocławku, autor artykułów z zakresu teologii moralnej fundamentalnej i szczegółowej. 


\section{LO SFORZO DI CAPONE PER UNA TEOLOGIA MORALE RINNOVATA}

Domenico Capone nei suoi scritti pone davanti ai nostri occhi il valore e la bellezza della vita morale cristiana (cioè della vita dell'uomo che ha provato l'esperienza dell'incontro con Cristo, che lo ha conosciuto e lo segue). Dall'insegnamento di Capone emerge il profumo del suo amore per Dio, per l'uomo e per la Chiesa.

Lattività teologica di Capone, professore dell'Accademia Alfonsiana a Roma, fu il frutto del grande entusiasmo rinnovatore della teologia durante i tempi conciliari e postconciliari, la chiamata al rinnovamento della teologia morale divenne la sua vocazione personale. Capone ebbe un influsso sulla riflessione postconciliare proponendo una morale lontana dal rigorismo e dalla casistica, fondandola, al contrario, sulla relazione personale dell'uomo con Cristo. La lettura di Capone ci fa capire come egli dal cristocentrismo trasse l'idea centrale per comprendere l'uomo nel suo essere ed esistere, connettendo l'uomo con Cristo: l'uomo è persona in Cristo ${ }^{1}$.

All'inizio della sua riflessione sta la domanda: "che cosè l'uomo?" e "che cosa costituisce l'uomo in quanto uomo?" La risposta è questa: "Crediamo che l'uomo se è persona, esistenzialmente non può esserlo se non in Cristo"2.

Siamo nel cuore della riflessione teologica di Capone. Il professore sviluppa la sua proposta teologica innestandola sulla visione cristocentrica, "solida base per una rinnovata teologia morale, alla luce del Concilio Vaticano II"3. Il centro della riflessione teologica rimane sempre l'uomo, ma nel suo rapportarsi a Cristo e all'altro. Le due dimensioni della vita umana costituiscono la realtà personale: la relazione con Cristo e la relazione con l'altro. La domanda su quale sia l'impatto della fede sull'agire della persona è la questione del rapporto tra l'esperienza dell'incontro con Cristo e la vita morale del credente. In questa luce, la vita morale dell'uomo ha a che fare con la costituzione della persona in Cristo. La teologia morale scopre come suo compito proprio il mostrare come l'incontro con Cristo influisca sull'agire della persona.

${ }^{1}$ Cfr. D. Capone, L'uomo è persona in Cristo, Bologna 1973, p. 22.

2 D. Capone, La coscienza morale, Dispensa 1974, p. 170.

${ }^{3}$ F. Parisi, Crisi e rinnovamento della teologia morale, Bologna 2013, p. 148. 


\section{STRUTTURA E DINAMISMI DELLA VITA MORALE CRISTIANA}

Capone esamina dunque gli elementi strutturali propri dellagire morale del cristiano che scaturiscono dall'affermazione centrale che l'uomo è persona in Cristo: la virtù della prudenza, il valore della coscienza cristiana, la fondazione cristologica e la dimensione pneumatologica e sacramentale della vita cristiana.

\section{a) la prudenza}

Secondo la visione di Capone, bisogna accentuare il ruolo della prudenza nella scoperta della verità morale. La prudenza deve essere illuminata dalla sapienza e rende sempre retta la ragione dell'azione morale. Dunque agire secondo la natura di persona, o secondo la retta ragione, significa agire secondo la prudenza che è "ministra" della sapienza. Proprio la prudenza compie la sintesi del bene trascendentale supremo con ciò che è in situazione concreta utile, interpretando così la scelta fatta dalla coscienza ${ }^{4}$. Riflettendo sulla virtù della prudenza, Capone afferma che anche la verità dell'atto morale proviene dall'unità dell'essere, dalla persona in Cristo.

La prudenza è la virtù nell'agire dell'uomo. In quanto virtù morale, Capone definisce la prudenza in questo modo: "La prudenza è virtù morale che, investita dalla luce e dalla forza della finalità che è presente nell'intenzione e nel giudizio intenzionale, cerca di conoscere bene il costitutivo obbiettivo dell'utilità, ma soprattutto valuta l'utilità dell'atto come si pone in concreto, nel soggetto" 5 .

Come viene notato, Capone subito aggiunge che la prudenza ha ruolo conoscitivo e direttivo. Quindi essa non è solo virtù morale bensì anche virtù intellettuale-morale, e aiuta a conoscere la verità così come è. Cerca dunque la verità oggettiva dell'atto da compiere come il mezzo più utile al raggiungere il fine ${ }^{6}$.

${ }^{4}$ Cfr. D. Capone, «La teologia della coscienza morale nel Concilio e dopo il Concilio», StMor 24 (1986), p. 227.

5 D. Capone, Intorno alla verità morale, Napoli 1951, p. 43.

${ }^{6}$ Cfr. ibid. 
Dal principio secondo cui la verità ultima dell'atto non viene dall'oggetto come è in sé, ma dall'oggetto come è nell'intenzione, la prudenza dice se l'atto è o non è attuazione interiore del fine così come è nell'intenzione retta. La prudenza conosce questa certezza dell'utilità dell'atto, perché essa mette in esistenza l'atto interiore secondo il giudizio della coscienza e garantisce la conformità del giudizio della coscienza con l'intenzione retta ${ }^{7}$.

La prudenza si presenta come maestra della sapienza. Essa deve assumere come principio e criterio di valore il mistero di Cristo. Latto principale della prudenza nello scegliere è la tensione verso Cristo ${ }^{8}$.

b) la coscienza

Relativamente al tema della coscienza bisogna ricordare la ricchezza del dibattito di quel periodo, presentando loriginale visione offerta da Capone basata sulla proposta di una fondazione cristologica della persona. Nei corsi tenuti all'Alfonsiana e in numerosi articoli "appaiono, in tutta la loro ampiezza e completezza, il valore e il significato della coscienza morale cristiana, compresi alla luce di GS 16; collocati all'interno e al centro di una rinnovata teologia morale come da OT 16". Capone esclude dunque una visione limitata e erronea della coscienza: non è una semplice singolarizzazione della norma di scienza con l'aggiunta della forza di obbligazione, oppure l'istanza indipendente dalla norma universale. Altri rischi nella comprensione della coscienza scaturiscono dal considerarla solamente di natura psicologica; dal relegarla in una posizione marginale rispetto all'intera teologia morale; dal darle una finalità essenzialmente applicativa e non come centro di ascolto ermeneuticamente creativo ${ }^{10}$. La posizione manualistica presente nel pensiero teologico fino al Concilio viene sostituita dalla nuova visione della coscienza morale cristiana alla luce del Vaticano II e di una fondazione cristiana della teologia morale basata sulla retta antropologia ${ }^{11}$.

\footnotetext{
7 Cfr. ibid., p. $45-46$.

${ }^{8}$ Cfr. ibid., p. 172.

9 F. Parisi, Crisi e rinnovamento, p. 163-164.

${ }^{10}$ Cfr. ibid., p. 166-167.

${ }^{11}$ Cfr. ibid., p. 167.
} 
Che cosè l'uomo? - risuona di nuovo la domanda fondamentale. Capone concentra la sua riflessione sulla coscienza proprio attorno alla questione antropologica, perché per lui l'essenza dell'uomo consiste nel suo agire da persona-immagine di Dio. Da quest'affermazione scaturisce l'altra: l'essere e l'agire come persona impongono un rapporto di interpersonalità e di dialogo nella chiave di chiamata - risposta. Si parla dunque della dialettica dell'incontro che si realizza nel sacrario intimo della coscienza dove Dio parla, Dio interpella e l'uomo è chiamato a dare una risposta secondo la grandezza della sua costituzione cristologica ad immagine di Dio in Cristo. La parola di questo dialogo è sempre Cristo - Parola di Dio. La coscienza dunque rivela la presenza personificante della Parola. Essa si presenta a noi come espressione e funzione della persona che ascolta e risponde, cioè dialoga con Dio, ma innanzitutto nella coscienza Dio e l'uomo si incontrano e costituiscono la comunione di vita ${ }^{12}$. La coscienza è la profondità dove l'incontro fondante si compie. Capone dice di più, la coscienza è dialogo filiale dell'uomo con il Padre" ${ }^{13}$. "La coscienza è il nucleo vitale in cui avviene l'incontro totale di Dio con la persona nella costruttività dell'amore"14, afferma Capone.

La precisa analisi della dispensa per il corso di morale fondamentale dell'anno accademico 1974-1975 dedicato interamente da Capone al tema della coscienza ci offre quest'approfondita proposta di una coscienza cristiana, cristicamente fondata, con gli accenti posti sullagire della persona (con la distinzione tra realtà naturale e sopranaturale), sulla realtà esistenziale del nostro essere e del nostro essere di persona, sull'intenzionalità e sulla opzione fondamentale, sulla prudenza nel deliberare in situazione e nell'attualizzare l'intenzionalità della persona, sulla dimensione cosmica e ultracosmica della salvezza operata da Cristo e alla fine sulla formazione della coscienza ${ }^{15}$.

${ }_{12}$ Cfr. D. Capone, «La coscienza come norma morale in situazione», $\mathrm{PrPa}$ 42 (1972), p. 140.

${ }^{13}$ Cfr. D. Capone, «S. Tommaso e S. Alfonso in teologia morale», Asp 21 (1974), p. 448.

${ }^{14}$ D. Capone, La coscienza morale, p. 54.

${ }^{15}$ Cfr. F. Parisi, Crisi e rinnovamento, p. 167-189. 
c) fondazione cristologica

Si passa alla fondazione cristologica della vita morale del cristiano. Gli elementi costitutivi di tale fondazione sono: "virtù della prudenza, che innervandosi di cristianesimo diventa virtù cardinale e assume un finalismo di natura intenzionale-sacramentale-escatologica, che coinvolge l'intero essere della persona e non solo l'aspetto filosofico o antropologico, per poi innestarsi decisamente sulla figura del Cristo, persona-proto-uomo, che personifica l'uomo, non solo restauratore dell'ordine morale, messo in crisi dal peccato di Adamo ed Eva, ma suo originario fondatore" ${ }^{16}$. Ricordiamo che per Capone ogni uomo è persona in Cristo.

Quali sono i seguenti elementi dell'essere persona in Cristo? Il primo è una premessa di natura antropologica: il nostro essere, esistere ed agire hanno una tensione di natura trascendentale - è la tensione dell'essere persona verso l'essere fontale che è Dio. Laltro è una verità di base: come spiega Capone, Cristo è in noi presente e operante in simbiosi reale, ontologica, che si attua con economia sacramentale; Egli non è soltanto oggetto di vita morale ma anche è soggetto, con-soggetto in noi, in piena simbiosi di essere, di esistere, di agire, in libertà di figli di Dio. Infine, una nuova visione della storia come meta-storia del Cristo risorto, cioè la storia della salvezza che salva la nostra storia personale ${ }^{17}$.

Da questo scaturisce la vera nozione del cristocentrismo in teologia morale secondo Capone. Secondo lui, Cristo in noi è principio di essere, esistere e agire. Questo ci offre immagini attive di Dio, in dialogo con Lui nella dialettica chiamata-risposta. Cristo semplicemente non rivela l'Idea di Dio, ma è anche pastore e salvatore dell'uomo. Cristo non è restauratore, ma fondatore originario della morale. La mediazione di Cristo esige una risposta a Dio data in Cristo perché Cristo dialoga con noi come parola del Padre e parola di sé, si dona a noi; noi nella comunione con Lui ed in Lui siamo parola di risposta al Padre. Tale risposta è da persona a persona perché il suo fondamento è la relazione interpersonale dell'uomo con la persona di Cristo $^{18}$.

\footnotetext{
16 Ibid., p. 190.

${ }^{17}$ Cfr. ibid., p. 196-197.

${ }^{18}$ Cfr. ibid., p. 198-200.
} 


\section{d) dimensione pneumatologica}

La dimensione pneumatologica e sacramentale della vita cristiana ci propone la domanda sulla santità. Capone, nei corsi di teologia morale fondamentale relativi alla teologia spirituale, sottolinea la carità in quanto via della sequela di Cristo e riscopre la presenza dinamica dello Spirito Santo nel nostro spirito attraverso la vita sacramentale, ma affronta soprattutto e direttamente il tema della santità, della vocazione generale alla santità. "Capone propone, alla luce del mistero del Cristo, una dimensione ontologica della santità, che si realizza nell'unione sacramentale con il Cristo «il solo Santo di Dio». Quindi il Cristo rimane l'unica via possibile per accedere alla santità che è fondamentalmente cristo-centrica e cristo-dinamica"19. Aprirsi alla santità significa dunque per Capone intensa partecipazione all'essere fontale di Cristo. Riferendosi allo Spirito Santo Capone supera definitivamente il legalismo o la casistica nella vita morale del cristiano.

\section{LA VITA MORALE CRISTIANA COME VITA DI UNIONE SACRAMENTALE CON CRISTO}

Nella tematica pastorale e sacramentale di Capone il principio guida della dimensione liturgico-sacramentale ed ecclesiale della morale è "la vita di unione sacramentale con il Cristo, che deve caratterizzare l'intera vita morale, specificamente cristiana" ${ }^{20}$. I sacramenti ci aiutano a diventare più simili a Dio e perciò buoni. Il sacramento "esprime questo status del Cristo in noi: parola intima di Gesù che è grazia e muove tutta la persona, che entra in sintonia con lui e tramite lui con tutta la Trinità"21. Inoltre, la realtà sacramentale si realizza sotto l'azione dello Spirito Santo, che pone in sintonia tutta la persona del cristiano con Cristo. In altre parole, essa ha la sua dimensione pneumatologica.

Per Capone "Cristo inizia la vita morale del cristiano attraverso il battesimo, la perfeziona nell'Eucaristia e «la corona in cielo trasforman-

\footnotetext{
19 Ibid., p. 206.

${ }^{20}$ Ibid., p. 226.

${ }^{21}$ Ibid., p. 211.
} 
doci da Signore nella sua umanità gloriosa di sacerdote eterno in seno alla vita paterna e trinitaria»" 22 . Nel sacramento del battesimo Cristo, che è quello della risurrezione, preleva l'uomo dalla morte e dal peccato. Nel sacramento della cresima si perfeziona l'azione dello Spirito Santo. Nell'Eucaristia avviene la trasformazione del cristiano nell'uomo-persona ontologicamente nuovo, in Cristo. Per il nostro autore tale impostazione della vita sacramentale fa sì che meglio si comprenda il dinamismo della chiamata-risposta: "la chiamata ci viene attraverso i sacramenti, genitori, la Chiesa; la risposta si pone come iniziale vita di fede-carità, quindi come orientamento totale verso Dio che chiama" ${ }^{23}$.

Affrontando il tema del sacramento della riconciliazione Capone propone una lettura non giuridica ma personalistica del sacramento. La riconciliazione ottenuta tramite la grazia sacramentale è come una nuova immersione in Cristo. Di nuovo, Cristo si pone come principio, valore e quindi come norma di vita all'interno della persona, come legge nuova che proclama la carità di Dio verso l'uomo e chiama l'uomo alla carità verso Dio e l'altro uomo. Non sorprende che in tale ottica l'uomo è sempre inteso come l'immagine di Dio; ciò significa che anche nel peccatore cè il riflesso dell'immagine di Dio. Nel sacramento della riconciliazione il valore da salvare è sempre la persona, perciò il compito del confessore si concretizza nel curare la persona del peccatore. A tutto questo basta aggiungere che il compito del confessore si può racchiudere in quattro parole: padre, medico, dottore, giudice. Tutto questo ci fa comprendere come la nuova morale proposta da Capone nella luce del Vaticano II chiede che il sacramento della riconciliazione sia celebrato e vissuto come incontro con Cristo e diventi così vera conversione al Cristo ${ }^{24}$.

In conclusione: un rapido sguardo al sacramento del matrimonio e alla famiglia cristiana rivela nuovamente la verità fondamentale per la vita morale di ogni cristiano. Il sacramento del matrimonio si poggia sul sacramento del battesimo, che pone gli sposi in comunione ontologicosacramentale con il Cristo e li fa partecipi del suo amore ${ }^{25}$. Gli sposi, grazie

22 D. Capone, Disegno di una teologia morale fondamentale, Dispensa 1968, p. 40.

${ }^{23}$ Ibid., p. 41.

${ }^{24}$ Cfr. F. Parisi, Crisi e rinnovamento, p. 220.

${ }^{25}$ Cfr. ibid., p. 222. 
al sacramento del matrimonio, hanno un nuovo modo di essere e diventano più simili a Cristo. Per questo i mezzi di vera cura della comunione coniugale sono la vita eucaristica con il sacramento della riconciliazione, la partecipazione alla vita liturgica della Chiesa e l'apostolato verso altre copie di sposi e verso le famiglie. Inoltre, occorre prendere in considerazione la dimensione personalistica per la spiritualità della famiglia: la famiglia è comunità di persone, fondata e costituita da comunione intima e unica che anche essa ha il suo fondamento nel Cristo $^{26}$.

\section{LA PROPOSTA MORALE DI DOMENICO CAPONE}

A cinquantanni dal concilio Vaticano II si può ribadire che per Capone una nuova morale, alla luce dei documenti conciliari è possibile e non più solo auspicabile ${ }^{27}$. Quali sono dunque i temi attorno ai quali si è sviluppato il pensiero di Domenico Capone ed è stata elaborata la sua proposta morale?

I primi temi riguardano il superamento della morale casistica ed i nuovi riferimenti culturali e filosofici. Nell'ambito della teologia morale però Capone indica e sviluppa la dimensione cristologica che contiene una specificità davvero innovativa, tale da superare la casistica e il legalismo, cioè essa racchiude un'ottica prudenziale ed è centrata sulla coscienza e sul Cristo redentore ${ }^{28}$. Capone, nel dibattito tra morale dei principi e morale della situazione (tra oggettivisti e soggettivisti) "operando sul processo decisionale, percepisce che il finalismo intenzionale che dirige l'azione del soggetto morale, sostenuto dalla virtù della prudenza, per il cristiano può essere rappresentato solo dalla carità del Cristo risorto. [...] Solo il Cristo è il nuovo principio assiologico di finalità per l’azione morale del cristiano" 29 .

In questa visione la figura del Cristo si presenta come rivelatore di Dio, in quanto persona, con l'essere proprio di Dio-persona-trinità, che

\footnotetext{
${ }^{26}$ Cfr. D. Capone, Per una teologia morale della famiglia, Dispensa 1982, p. 34.

27 Cfr. F. Parisi, Crisi e rinnovamento, p. 229.

${ }^{28}$ Cfr. ibid., p. 235.

${ }^{29}$ Cfr. ibid.
} 
crea l'uomo, chiamandolo a vivere una relazione più intima, nella comunione con sé; un Cristo che nei vangeli si presenta come rivelazione dellessenza amorosa di Dio e della sua dimensione trinitaria e che perfeziona la sua opera di salvezza con l'opera dello Spirito Santo. Di conseguenza, l'uomo si presenta come volontà cosciente e libera e soggetto responsabile di moralità, padrone del proprio atto, con Cristo-con-soggetto della stessa azione morale. Si tratta dell'uomo che è persona in $\mathrm{Cristo}^{30}$.

In conseguenza l'uomo è chiamato a superare il minimalismo morale e realizzare la vocazione alla santità. Parisi conferma: "il cammino di santità, per tutti i cristiani, prende avvio dall'incontro con Cristo, fonte di ogni santità, si traduce in sequela, si ritrova in intimità, per Cristo in Dio, nel sacrario della coscienza, si alimenta di vita liturgica e sacramentale, si confronta con la comunità ecclesiale e i suoi pastori, si perfeziona nella carità e si sviluppa in una storia (escatologica) di salvezza" ${ }^{31}$. Il progetto di rinnovamento della teologia morale dunque ha in Capone un'immediata ricaduta pastorale; esso non è una teoria bensì la proposta per ciascuno di noi che portiamo il nome dei cristiani.

Durante il congresso "Camminare nella luce. Prospettive della teologia morale a 10 anni dalla Veritatis Splendor" svoltosi a Roma presso l'Università Lateranense nel 2004, il prefetto di allora della Congregazione per la Dottrina della Fede, card. Joseph Ratzinger durante il suo discorso, dichiarando che la Veritatis Splendor intendeva di riprendere e riproporre il Concilio Vaticano II nel suo messaggio morale, ha affermato che la Gaudium et spes voleva "ritornare ad una morale sostanzialmente biblica e cristologica, ispirata dall'incontro con Cristo, una morale non concepita come una serie di precetti ma come l'avvenimento di un incontro, di un amore che poi sa creare anche azioni corrispondenti" ${ }^{2}$. In quest'affermazione echeggiano già i temi principali della teologia di Capone e della sua impostazione secondo lo spirito del Vaticano II. Ratzinger ha inoltre descritto la situazione postconciliare, del tutto imprevedibile, dove anziché rinnovare una teologia morale di ispirazione biblica, questi tentativi si

${ }^{30}$ Cfr. ibid., p. 236-237.

${ }^{31}$ Cfr. ibid., p. 239.

32 J. Ratzinger, Il rinnovamento della teologia morale: prospettive del Vaticano II e di Veritatis splendor, [in:] Camminare nella luce. Prospettive della teologia morale a partire da Veritatis splendor, ed. L. Melina, J. Noriega, Roma 2004, p. 36. 
sono fermati, senza portare alla nuova primavera per una teologia morale profondamente cristologica e biblica. Secondo Ratzinger i tentativi dei teologi hanno portato la marginalizzazione della Sacra Scrittura. Di conseguenza, il Cardinale ha ribadito: "Guardando al modo in cui fu recepita l'enciclica Veritatis splendor, la mia delusione non derivò tanto dal fatto che essa diede occasione a molte critiche, ma piuttosto dal fatto che non si sia entrati in questo grande dibattito circa i principi della morale, circa questa grande e rinnovata visione nello stesso tempo cristologica e razionale, perché Cristo è il Logos"33. In questo contesto possiamo vedere che la visione di Capone è quanto mai attuale, è da realizzare e da riprendere. Senza rischio si può affermare che la proposta morale di Capone è profetica in relazione alla tradizione morale della Chiesa di oggi. La voce di Ratzinger è proprio il punto di riferimento per tale constatazione. La via del rinnovamento della teologia morale postconciliare, così come l'ha proposta Capone, è la via del ritorno allo spirito del Vaticano II nell'insegnamento morale della Chiesa di oggi.

Per esempio, nell'insegnamento di Giovanni Paolo II nella Veritatis Splendor, viene riconosciuta la centralità della figura di Cristo che implica la vera riconciliazione tra storia e ragione, tra rivelazione soprannaturale e ragione perché Cristo è il Logos fatto carne. L'uomo è una persona che vive in forza di una dipendenza, che in realtà significa la relazione dell'amore e la vera forma di partecipazione all'essere di Dio (nel linguaggio di Capone si potrebbe dire: "all'essere fontale"). Vivere nella comunione con Dio e trovare il vero cammino, trovarvi la via, la verità e la vita significa ritrovare se stesso nella propria vera identità ${ }^{34}$. La visione cristologica dell'uomo ci dice che l'uomo entrando nella vera comunione con se stesso trova la sua vera essenza e raggiunge la sua vera libertà. Giovanni Paolo II indica le vie della perfezione dell'uomo affermando, che "seguire Cristo è il fondamento essenziale e originale della morale cristiana" (VS 19). La vita morale invece si presenta come risposta dovuta alle iniziative gratuite che lamore di Dio moltiplica nei confronti dell'uomo. È una risposta d'amore di Dio ed è chiamata a riflettere la gloria di Dio. Seguire Cristo significa aderire alla persona stessa di Gesù e condividere la sua

\footnotetext{
${ }^{33}$ Ibid., p. 42.

${ }^{34}$ Cfr. ibid., p. 43.
} 
vita e il suo destino (VS 19), amare di un amore che si dona totalmente ai fratelli per amore di Dio (VS 20), imitare Cristo, assimilandosi a Lui (VS 21). La sequela di Cristo in altre parole è la vocazione alla santità. E Giovanni Paolo II ha insegnato che martirio è il frutto più maturo della sequela di Cristo (VS 90).

Anche papa Benedetto XVI ha approfondito la visione già presentata del rinnovamento della morale cristiana. Soprattutto nell'enciclica Deus caritas est ha scritto di Gesù Cristo come l'amore incarnato di Dio. La vita morale è la vita di ciascuno che ha l'esperienza dell'amore di Dio in Gesù Cristo, che vive l'esperienza dell'incontro con Dio. In questo incontro si rivela l'amore di Dio: "Il riconoscimento del Dio vivente è una via verso l'amore [...] Questo però è un processo che rimane continuamente in cammino: l'amore non è mai concluso e completato; si trasforma nel corso della vita, matura e proprio per questo rimane fedele a se stesso" (DCE 17).

Ricordiamo inoltre le prime omelie di papa Francesco dove egli ci ha chiamato al camminare nella presenza del Signore, "cercando di vivere con quella irreprensibilità che Dio chiedeva ad Abramo, nella sua promessa. [...] Io vorrei che tutti abbiamo il coraggio di camminare nella presenza del Signore". Nella sua esortazione Francesco afferma che "lamore di Gesù che abbiamo ricevuto, l'esperienza di essere salvati da Lui, ci spinge ad amarlo sempre di più [...] Non è la stessa cosa aver conosciuto Gesù o non conoscerlo, non è la stessa cosa camminare con Lui o camminare a tentoni, non è la stessa cosa poterlo ascoltare o ignorare la sua Parola, non è la stessa cosa poterlo contemplare, adorare, riposare in Lui, o non poterlo fare" (Evangelii Gaudium 264.266). Questi accenni ci fanno subito capire che vale la pena riscoprire la proposta morale di Domenico Capone davanti alle sfide della vita di oggi.

Streszczenie. Refleksja na temat chrześcijańskiego życia moralnego. Propozycja moralna Domenico Capone 50 lat po Soborze Watykańskim II. 50 lat po zakończeniu Soboru Watykańskiego II, w niniejszym artykule zaproponowane zostało spojrzenie na pasjonującą teologiczną drogę profesora Akademii Teologii Moralnej w Rzymie (Accademia Alfonsiana) Domenico Capone, który przedstawił propozycję moralną aktualną dla człowieka XXI wieku. W jakimś sensie to, co zaproponował Capone, było wizją proroczą na obecne czasy, w jakich Kościół rozwija swoje nauczanie. Droga odnowy życia moralnego zaproponowana przez niego opiera się na refleksji nad człowiekiem, 
jego działaniem i życiem moralnym. Ten wielki teolog rozumiał człowieka jako „osobę w Chrystusie”, a więc jako tego, który doświadczył spotkania z Chrystusem w swoim życiu, poznał Go i wszedł na drogę Jego naśladowania. Z tego doświadczenia bierze się struktura i dynamizm życia moralnego i na nim opiera się cała odnowa chrześcijańskiej moralności zaproponowana przez Domenico Capone: chrystologiczny fundament życia moralnego chrześcijanina, dzięki któremu Chrystus staje się w nas zasadą egzystencji i działania, wezwanie i odpowiedź jako elementy dialogicznej natury relacji człowieka z Bogiem, upodobnienie się do Chrystusa, realizacja powołania do życia w miłości, co w konsekwencji prowadzi do codziennego dawania odpowiedzi życiem na powołanie do świętości. Taki model chrześcijańskiej moralności, zaproponowany już ponad pół wieku temu, znalazł swoje odzwierciedlenie $\mathrm{w}$ dokumentach soborowych oraz $\mathrm{w}$ nauczaniu papieży: św. Jana Pawła II, Benedykta XVI i Franciszka, co czyni propozycję moralną Domenico Capone jak najbardziej aktualną.

Słowa kluczowe: życie moralne; Domenico Capone; Sobór Watykański II.

Abstract. Reflect on the Christian moral life. The moral proposal by Dominic Capone 50 years after the Vatican Second Council. Fifty years after the end of the Second Vatican Council, this article was proposed to look at the exciting theological road of one of the professors of the Academy of Moral Theology in Rome (Accademia Alfonsiana), Domenico Capone, who proposed a vision of moral theology for the twenty first century. In a sense, what Capone suggested, was a prophetic vision for the present times, in which the Church develops her teaching. A way of renewal of the moral life proposed by Capone is based on a reflection on man's action and the moral life. The great theologian understood man as ,a person in Christ”, so as one who has experienced the encounter with Christ in his life, he met him, and decided to follow Him. From this experience comes the structure and dynamism of the moral life and on it rests the whole renewal of Christian morality proposed by Domenico Capone: the Christological foundation of the moral life of a Christian, by which Christ becomes in us the principle of existence and activities, call and response as part of the dialogic nature of the relationship of man and God, become similar to Christ, fulfillment of the vocation to live in love, what leads to give an answer to the call to holiness in everyday life. Such a model of Christian morality proposed more than half a century ago was reflected in the documents of the Vatican II as well as in the teaching of the popes: St. John Paul II, Benedict XVI and Francis, a fact that makes Domenico Capone's moral proposal the most current.

Key words: moral life; Domenico Capone; Vaticanum II. 
